\title{
THE FIRST PONTRJAGIN CLASSES OF HOMOTOPY COMPLEX PROJECTIVE SPACES
}

\author{
YASUHIKO KITADA AND MAKI NAGURA
}

\begin{abstract}
Let $M^{2 n}$ be a closed smooth manifold homotopy equivalent to the complex projective space $\mathbb{C} P(n)$. The purpose of this paper is to show that when $n$ is even, the difference of the first Pontrjagin classes between $M^{2 n}$ and $\mathbb{C} P(n)$ is divisible by 16 .
\end{abstract}

\section{INTRODUCTION AND THE MAIN THEOREM}

Let $M^{2 n}$ be a closed smooth manifold and $\mathbb{C} P(n)$ be the complex projective space of complex dimension $n$. If there is a homotopy equivalence $f: M^{2 n} \rightarrow \mathbb{C} P(n)$, we say that $M^{2 n}$ is a homotopy projective space or more briefly a homotopy $\mathbb{C} P(n)$. When $M^{2 n}$ is a homotopy projective space and $f: M^{2 n} \rightarrow \mathbb{C} P(n)$ is a homotopy equivalence, define an integer $\delta(M)$ by $p_{1}(M)-f^{*}\left(p_{1}(\mathbb{C} P(n))\right)=\delta(M) u^{2}$, where $p_{1}(M)$ is the first Pontrjagin class of $M^{2 n}$ and $u$ is a generator of $H^{2}\left(M^{2 n} ; \mathbb{Z}\right)$. Clearly $\delta(\mathbb{C} P(n))$ is zero and $\delta(M)$ measures the difference between the first Pontrjagin classes of the two manifolds $M^{2 n}$ and $\mathbb{C} P(n)$. When $n=3$, Montgomery and Yang studied and classified homotopy complex projective spaces $M^{6}$ and proved that the first Pontrjagin class $p_{1}(M)$ is of the form $p_{1}\left(M^{6}\right)=(4+24 \alpha(M)) u^{2}$ for some integer $\alpha(M)$ where $u \in H^{2}\left(M^{6} ; \mathbb{Z}\right)$ is a generator. They also showed that the diffeomorphism type of a homotopy $\mathbb{C} P(3)$ is determined by the first Pontrjagin class ([1] p.25, 7], 8] Theorem A). In general dimensions, Masuda and Tsai proved that for a homotopy complex projective space $M^{2 n}, \delta\left(M^{2 n}\right)$ is divisible by 24 (6] Lemma 5.1). In 1971, Brumfiel calculated index surgery obstructions with target $\mathbb{C} P(4)$ and $\mathbb{C} P(6)$ and reported that $\delta\left(M^{2 n}\right)$ is divisible by 16 for $n=4$ and $n=6$ ([2] Lemma I.5).

Brumfiel's calculation was based on the calculation of the cohomology group of the classifying space $G / O$. But the details of his calculation are not published. So we took one step a way from the surgery theory and looked closely at Hirzebruch's index theorem. We were able to obtain our final result in the following theorem.

Main Theorem. Let $M^{4 k}$ be a homotopy $\mathbb{C} P(2 k)$. Then $\delta\left(M^{4 k}\right)$ is divisible by 16.

In the previous work of the first author [4, there was a restriction on the 2-order of the integer $k$. In the present paper, this restriction is completely removed.

\section{Preliminaries: formal POWER SERIES AND Elementary Number theOry}

We shall consider the ring $\mathbb{Q}[[x]]$ of formal power series with rational coefficients. An element $f(x) \in \mathbb{Q}[[x]]$ can be written as

$$
f(x)=\sum_{i \geq 0} c_{i} x^{i}, \quad\left(c_{i} \in \mathbb{Q}\right) .
$$

2000 Mathematics Subject Classification. 57R20, 57R55, 57R67. 
If $f(x)$ is not 0 , then there exists a non-negative integer $j$ such that $c_{i}=0$ for all $i<j$ and $c_{j} \neq 0$. This number $j$ is the order of $f(x)$ and expressed by $\operatorname{ord}(f(x))$. If $\rho=\operatorname{ord}(f(x))$, then $f(x)$ can be expressed as

$$
f(x)=x^{\rho}\left(c_{\rho}+c_{\rho+1} x+c_{\rho+2} x^{2}+\cdots\right) .
$$

From this we see that the quotient field of $\mathbb{Q}[[x]]$ is the ring of formal Laurent series

$$
F(x)=\sum_{i \geq r} c_{i} x^{i}
$$

where $c_{i} \in \mathbb{Q}$ and $r \in \mathbb{Z}$, with only a finite number of negative degree terms. We shall simply call this expression a formal Laurent series. Given a formal Laurent series $F(x)$, we shall denote the coefficient of $x^{i}$ in $F(x)$ by $(F(x))_{i}$ or by $(F(x))_{x^{i}}$. The latter notation is usually used when we want to specify the variable $x$. The coefficient of $x^{-1}$ is called the formal residue of the formal Laurent series $F(x)$ and often denoted by $\operatorname{Res}_{x}(F(x))$.

Let $G(y)=\sum_{i \geq 0} r_{i} y^{i}$ be a formal power series with $r_{0}=G(0)=0$. Then for any formal Laurent series $F(x)$, we can perform substitution $x=G(y)$ to obtain a new formal Laurent series $F(G(y))$ with variable $y$. This series is expressed by $F \circ G$. When $F(x)$ is a formal power series, then $F \circ G$ is also a formal power series. Corresponding to the substitution, we have the invariance of formal residues is given by the following lemma.

Lemma 1. Let $F(x)=\sum_{i} q_{i} x^{i}$ be a formal Laurent series and $G(y)=\sum_{i} r_{i} y^{i}$ be a formal power series with $r_{0}=G(0)=0$ and $r_{1} \neq 0$. Then we have

$$
\operatorname{Res}_{x}(F(x))=\operatorname{Res}_{y}\left(F(G(y)) G^{\prime}(y)\right),
$$

where $G^{\prime}(y)=\sum_{i}(i+1) r_{i+1} y^{i}$ is the formal derivative of $G(y)$.

Proof. Since a formal Laurent series is a linear combination of $x^{n}(n \in \mathbb{Z})$, it is enough to show the formula for the special case $F(x)=x^{n}$. Unless $n=-1$, since $F(G(y)) G^{\prime}(y)=G(y)^{n} G^{\prime}(Y)$ is a formal derivative of $G(y)^{n+1} /(n+1)$, its residue with respect to the variable $y$ is zero. Thus the formula holds for $F(x)=x^{n}$ with $n \neq-1$. For the case $n=-1$, we have

$$
\begin{aligned}
F(G(y)) G^{\prime}(y) & =\frac{G^{\prime}(y)}{G(y)}=\frac{\sum_{i} i r_{i} y^{i-1}}{\sum_{i} r_{i} y^{i}} \\
& =\frac{\sum_{i \geq 1} i r_{i} y^{i-1}}{y \sum_{i \geq 1} r_{i} y^{i-1}}=\frac{H(y)}{y}
\end{aligned}
$$

for some formal power series $H(y)=\sum_{i \geq 0} c_{i} y^{i}$ with $c_{0}=1$. Therefore we have

$$
\operatorname{Res}_{y}\left(G^{\prime}(y) / G(y)\right)=1 \text {. }
$$

This completes the proof.

For a prime $p, \mathbb{Z}_{(p)}$ is a subring of $\mathbb{Q}$ composed of rational numbers that can be expressed as $a / b(a, b \in \mathbb{Z}, b \neq 0)$, with $(b, p)=1$. Its invertible element is of the form $a / b$ with $(a, p)=1$ and $(b, p)=1$. If the coefficients of $F(x)$ and $G(y)$ are in $\mathbb{Z}_{(p)}$, and if in addition $G^{\prime}(0)$ is an invertible element, then the substitutions and the formula (1) in Lemma 1 can be performed in the same coefficient ring $\mathbb{Z}_{(p)}$. The following is an inverse function theorem in the formal power series theory.

Lemma 2. Let $p$ be a prime and $F(x)=\sum_{i>0} q_{i} x^{i} \in \mathbb{Z}_{(p)}[[x]]$ be a formal power series and assume that $F(0)=q_{0}=0$ and $F^{\prime}(0)=q_{1}$ is invertible in $\mathbb{Z}_{(p)}$. Then there exists a unique formal power series $G(y)$ in $\mathbb{Z}_{(p)}[[y]]$ with $G(0)=0$ such that $F(G(y))=y$. 
Proof. Let us write $G(y)=\sum_{i>0} r_{i} y^{i}$. Then we shall show that the coefficients $\left\{r_{i}\right\}$ of $G(y)$ are inductively uniquely determined as an element of $\mathbb{Z}_{(p)}$, by the equality $F(G(y))=y$ starting from the initial condition $G(0)=r_{0}=0$. Let us write $(G(y))^{i}=\sum_{j} r_{j}^{(i)} y^{j}$ then we can easy see that $r_{j}^{(i)}=0$ for $j<i$. From the equality $F(G(y))=y$, we have $\sum_{i} q_{i}\left(\sum_{j} r_{j}^{(i)} y^{j}\right)=y$. This reduces to

$$
\sum_{i}\left(\sum_{i \leq j} q_{i} r_{j}^{(i)}\right) y^{j}=y \text {. }
$$

From this we have $q_{1} r_{1}^{(1)}=1$. Since $q_{1}$ is invertible, $r_{1}=r_{1}^{(1)} \in \mathbb{Z}_{(p)}$ is determined. For $j \geq 2$, we have

$$
\sum_{i=1}^{j} q_{i} r_{j}^{(i)}=q_{1} r_{j}^{(1)}+q_{2} r_{j}^{(2)}+\cdots+q_{j} r_{j}^{(j)}=0 .
$$

If $r_{1}, r_{2}, \ldots, r_{j-1}$ are determined as elements of $\mathbb{Z}_{(p)}$, then $r_{1}^{(i)}, r_{2}^{(i)}, \ldots, r_{j}^{(i)}$ are determined in $\mathbb{Z}_{(p)}$ for $1<i \leq j$ as the coefficients of the polynomial

$$
\left(r_{1} y+r_{2} y^{2}+\cdots+r_{j-1} y^{j-1}\right)^{i} .
$$

This shows that $r_{j}^{(2)}, r_{j}^{(3)}, \ldots, r_{j}^{(j)}$ are determined and from (2), $r_{j}=r_{j}^{(1)}$ is determined as an element of $\mathbb{Z}_{(p)}$.

Let $\mathbb{Z}_{(p)}[[x]]_{1}$ stand for the subset of all formal power series with coefficients in the ring $\mathbb{Z}_{(p)}[[x]]$, with constant term 1 . As a corollary to the inverse function theorem we have a generalized binomial expansion formula.

Corollary 3. Let $p$ be a prime and $q$ be a natural number relatively prime to $p$. Then there exists a unique formal power series $v(x) \in \mathbb{Z}_{(p)}[[x]]_{1}$ satisfying $(v(x))^{q}=$ $1+x$.

Proof. Consider the formal power series $F(x)=(1+x)^{q}-1$. Then we have $F(0)=0$ and $F^{\prime}(0)=q$ is invertible in $\mathbb{Z}_{(p)}$. By Lemma 2, there exists a unique formal power series $G(y) \in \mathbb{Z}_{(p)}[[y]]$ satisfying $G(0)=0$ and $(1+G(y))^{q}-1=y$. If we put $v(x)=1+G(x)$, we see that $v(x)^{q}=1+x$.

We shall denote the formal power series $1+G(x)$ in the proof above by $(1+x)^{1 / q}$. It is well known that for a rational number $\alpha$, we have a formal power series expansion

$$
(1+x)^{\alpha}=1+\sum_{i \geq 1}\left(\begin{array}{c}
\alpha \\
i
\end{array}\right) x^{i},
$$

where $\left(\begin{array}{c}\alpha \\ i\end{array}\right)=\alpha(\alpha-1) \cdots(\alpha-i+1) / i$ !. When $\alpha=1 / q$ where $q$ is an integer prime to $p$, then from the corollary above, the coefficients $\left(\begin{array}{c}\alpha \\ i\end{array}\right)$ belong to $\mathbb{Z}_{(p)}$. This is also true for general $\alpha \in \mathbb{Z}_{(p)}$.

Proposition 4. Let $p$ be a prime, and $f(x) \in \mathbb{Z}_{(p)}[[x]]_{1}$. For a natural number $q$ such that $(p, q)=1$, there exists a unique formal power series $\varphi(x) \in \mathbb{Z}_{(p)}[[x]]_{1}$ that satisfies $(\varphi(x))^{q}=f(x)$.

Proof. From Corollary 3, there exists $v(x) \in \mathbb{Z}_{(p)}$ satisfying $(v(x))^{q}=1+x$. Substituting the variable $x$ by $f(x)-1$ in $v(x)$ we obtain $\varphi(x)=v(f(x)-1)$. Then we have $(\varphi(x))^{q}=1+(f(x)-1)=f(x)$. The uniqueness of such $\varphi(x)$ can be shown by the inductive argument as in the proof of Lemma 1 . 
Corollary 5. Let $p$ be a prime and let $\alpha=m / q, m, q \in \mathbb{Z}$ with $(p, q)=1$. Then for any $f(x) \in \mathbb{Z}_{(p)}[[x]]_{1}$, then the following formula holds in $\mathbb{Z}_{(p)}[[x]]_{1}$ :

$$
(f(x))^{m / q}=\left(f(x)^{1 / q}\right)^{m}=\left(f(x)^{m}\right)^{1 / q} .
$$

Next we shall introduce the notations that will frequently appear in this paper and explain number theoretic facts which will be used in the proofs. Let us fix a prime number $p$. For any integer $n$ the $p$-order of $n$ is the exponent of $p$ in the prime factorization of $n$ and is denoted by $\nu_{p}(n)$. By convention, we set $\nu_{p}(0)=\infty$. For a rational number $m / n$, where $m, n \in \mathbb{Z}$, we define $\nu_{p}(m / n)=\nu_{p}(m)-\nu_{p}(n)$. In the $p$-ary notation of a nonnegative integer $n=\sum_{i} n_{i} p^{i}$, the sum of digits $\sum_{i} n_{i}$ is denoted by $\kappa_{p}(n)$.

Here we present a fundamental lemma in treating the $p$-order of the coefficients of powers of a sum.

Lemma 6. Let $p$ be a prime and $m, n$ be non-negative integers.

(a) For variables $x$ and $y$, we have

$$
(x+y)^{p^{m+n}} \equiv\left(x^{p^{m}}+y^{p^{m}}\right)^{p^{n}} \bmod p^{n+1} .
$$

(b) For variables $x_{1}, x_{2}, \ldots, x_{r}$, we have

$$
\left(x_{1}+x_{2}+\cdots+x_{r}\right)^{p^{m+n}} \equiv\left(x_{1}^{p^{m}}+x_{2}^{p^{m}}+\cdots+x_{r}^{p^{m}}\right)^{p^{n}} \bmod p^{n+1} .
$$

Proof. To prove (a) we use induction on $n$. It is well known that the assertion is true for $n=0$. Assume that (a) is true for $n$. Then we can write

$$
(x+y)^{p^{m+n}}=\left(x^{p^{m}}+y^{p^{m}}\right)^{p^{n}}+p^{n+1} L
$$

for some $L \in \mathbb{Z}[x, y]$. Then taking the $p$-th power, we have

$$
\begin{aligned}
(x+y)^{p^{m+n+1}} & =\left(x^{p^{m}}+y^{p^{m}}\right)^{p^{n+1}}+\sum_{i=1}^{p}\left(\begin{array}{c}
p \\
i
\end{array}\right)\left(x^{p^{m}}+y^{p^{m}}\right)^{p^{n}(p-i)}\left(p^{n+1} L\right)^{i} \\
& =\left(x^{p^{m}}+y^{p^{m}}\right)^{p^{n+1}} \bmod p^{n+2} .
\end{aligned}
$$

This shows that the assertion is also true for $n+1$. The proof of (b) goes similarly using the induction on $n$ and is omitted.

From this lemma, we have the following formula.

Corollary 7. Given a formal power series $f(x) \in \mathbb{Z}_{(p)}[[x]]$. If $p$ is a prime, we have

$$
(f(x))^{p^{m+n}} \equiv\left(\sum_{i \geq 0}\left(c_{i} x^{i}\right)^{p^{m}}\right)^{p^{n}} \bmod p^{n+1} .
$$

Corollary 8. Let $p$ be a prime and a formal power series $f(x)=\sum_{i \geq 0} c_{i} x^{i}$, let us express its l-th power as

$$
(f(x))^{l}=\sum_{i \geq 0} c_{i}^{(l)} x^{i}
$$

If all the coefficents of $f(x)$ are in $\mathbb{Z}_{(p)}$, then we have

$$
\nu_{p}\left(c_{i}^{(l)}\right) \geq \nu_{p}(l)-\nu_{p}(i) .
$$

Proof. Let us fix $i$. The assertion trivially holds if $\nu_{p}(l) \leq \nu_{p}(i)$. So we may assume that $\nu_{p}(l) \geq \nu_{p}(i)+1$. We set $m=\nu_{p}(i)+1$ and $n=\nu_{p}(l)-m$. Then since $m+n=\nu_{p}(l)$, we can write $l=p^{m+n} q$ for some $q \in \mathbb{Z}_{+}$with $(p, q)=1$. From Corollary 7 , we have

$$
(f(x))^{l}=(f(x))^{p^{m+n} q} \equiv\left(\sum_{\substack{j \geq 0 \\ 4}}\left(c_{j} x^{j}\right)^{p^{m}}\right)^{p^{n} q} \bmod p^{n+1} .
$$


This shows that since $i$ is not divisible by $p^{m}, c_{i}^{(l)} \equiv 0 \bmod p^{n+1}$ holds. That is

$$
\nu_{p}\left(c_{i}^{(l)}\right) \geq n+1=\nu_{p}(l)-m+1=\nu_{p}(l)-\nu_{p}(i) .
$$

In this paper we are interested in the case where $p=2$ and we shall only consider the case $p=2$ from now on.

Lemma 9. Let $n, k$ be integers with $0 \leq k \leq n$. Then we have the following.

(a) $\nu_{2}(n !)=n-\kappa_{2}(n)$.

(b) $\quad \nu_{2}\left(\left(\begin{array}{l}n \\ k\end{array}\right)\right)=\kappa_{2}(k)+\kappa_{2}(n-k)-\kappa_{2}(n)$.

(c) Let $n=\sum_{i} n_{i} 2^{i}, \quad k=\sum_{i} k_{i} 2^{i}$ be the binary notations of $n$ and $k$. Then the binomial coefficient $\left(\begin{array}{l}n \\ k\end{array}\right)$ is even if and only if there exists $i$ such that $n_{i}<k_{i}$.

Proof. It is not difficult to see that the two sequences $\left\{q_{n}^{(i)}\right\},(i=1,2)$ defined by $q_{n}^{(1)}=\nu_{2}(n !)$ and by $q_{n}^{(2)}=n-\kappa_{2}(n)$ both satisfy the same inductive formula

$$
q_{0}^{(i)}=0, \quad q_{n}^{(i)}=[n / 2]+q_{[n / 2]}^{(i)},
$$

where $[t]$ denotes the largest integer not exceeding $t$. This formula uniquely determines the sequences $\left\{q_{n}^{(i)}\right\}$ and this fact proves (a). (b) follows immediately from (a). To show (c), if there exists a column $i$ such that $n_{i}<k_{i}$ then in the addition process of $k$ and $n-k$ in binary form, there exists a column where the digit addition carries 1 to the next column. If such a column exists, there arises a decrease of sum of digits as in

$$
\kappa_{2}(k)+\kappa_{2}(n-k)>\kappa_{2}(n)
$$

and this proves our assertion.

Lemma 10. Let $i, n$ and $m$ be natural numbers and assume that $n$ and $m$ are odd. Then we have the following.
(a) $\nu_{2}\left(n^{m}+1\right)=\nu_{2}(n+1) \quad$ and $\quad \nu_{2}\left(n^{m}-1\right)=\nu_{2}(n-1)$.
(b) $\nu_{2}\left(n^{2 i}-1\right)=\nu_{2}\left(n^{2}-1\right)+\nu_{2}(i)$.
(c) $\nu_{2}\left(n^{i}-(-1)^{i}\right)= \begin{cases}\nu_{2}(n+1), & \text { if } i \text { is odd } \\ \nu_{2}\left(n^{2}-1\right)+\nu_{2}(i)-1, & \text { if } i \text { is even. }\end{cases}$

Proof. (a) follows immediately from the factorizations

$$
\begin{aligned}
& n^{m}+1=(n+1)\left(n^{m-1}-n^{m-2}+\cdots-n+1\right) \\
& n^{m}-1=(n-1)\left(n^{m-1}+n^{m-2}+\cdots+n+1\right) .
\end{aligned}
$$

To show (b), in view of (a) we may assume, without loss of generality, that $i=2^{e}$. Then from the factorization

$$
n^{2 i}-1=\left(n^{2}-1\right)\left(n^{2}+1\right)\left(n^{2^{2}}+1\right) \cdots\left(n^{2^{e}}+1\right),
$$

and from the fact that $\nu_{2}\left(n^{2^{r}}+1\right)=1$ for $r \geq 1$, we have the conclusion by (a) and (b).

Lemma 11. Let $i_{1}, i_{2}, \ldots, i_{s}$ be non-negative integers.

(a) If $i_{1} \geq 1$, then

$$
\nu_{2}\left(i_{1}\right)+\kappa_{2}\left(i_{1}\right)+\kappa_{2}\left(i_{2}\right) \geq \nu_{2}\left(i_{1}+i_{2}\right)+1 .
$$

(b) If $i_{1} \geq 1$, then

$$
\nu_{2}\left(i_{1}\right)+\kappa_{2}\left(i_{1}\right)+\kappa_{2}\left(i_{2}\right)+\cdots+\kappa_{2}\left(i_{s}\right) \geq \nu_{2}\left(i_{1}+\cdots+i_{s}\right)+1 .
$$


Proof. The proof of (a) is divided into several cases.

Case : $i_{1}+i_{2}$ odd. Then since $\kappa_{2}\left(i_{1}\right) \geq 1$ and $\nu_{2}\left(i_{1}+i_{2}\right)=0$, we get the assertion. Case: both $i_{1}$ and $i_{2}$ odd. Express $i_{1}$ and $i_{2}$ in the binary notations:

$$
i_{1}=\sum_{j \geq 0} s_{j} 2^{j}, \quad i_{2}=\sum_{j \geq 0} t_{j} 2^{j}
$$

where $s_{j}, t_{j}=0,1$. Let $d=\nu_{2}\left(i_{1}+i_{2}\right)$ then we have $s_{0}=t_{0}=1$, and for each $j$ with $1 \leq j \leq d-1, s_{j}+t_{j}=1$. From this we have

$$
\nu_{2}\left(i_{1}\right)+\kappa_{2}\left(i_{1}\right)+\kappa_{2}\left(i_{2}\right) \geq d+1=\nu_{2}\left(i_{1}+i_{2}\right)+1 .
$$

Case : both $i_{1}$ and $i_{2}$ even. Let $t=\min \left(\nu_{2}\left(i_{1}\right), \nu_{2}\left(i_{2}\right)\right)$. Then we can write $i_{1}=2^{t} j_{1}$, $i_{2}=2^{t} j_{2}$ and $j_{1}$ or $j_{2}$ is odd. Therefore we have

$$
\nu_{2}\left(j_{1}\right)+\kappa_{2}\left(j_{1}\right)+\kappa_{2}\left(j_{2}\right) \geq \nu_{2}\left(j_{1}+j_{2}\right)+1 .
$$

Adding $t$ to both sides, we have

$$
\nu_{2}\left(i_{1}\right)+\kappa_{2}\left(i_{1}\right)+\kappa_{2}\left(i_{2}\right) \geq \nu_{2}\left(i_{1}+i_{2}\right)+1 .
$$

This completes the proof of (a).

(b) follows immediately using (a) as follows:

$\nu_{2}\left(i_{1}\right)+\kappa_{2}\left(i_{1}\right)+\cdots+\kappa_{2}\left(i_{s}\right) \geq \nu_{2}\left(i_{1}\right)+\kappa_{2}\left(i_{1}\right)+\kappa_{2}\left(i_{2}+\cdots+i_{s}\right) \geq \nu_{2}\left(i_{1}+\cdots+i_{s}\right)+1$.

Now we shall present definitions, notations and basic facts about Bernoulli numbers and Hirzebruch power series. Recall that the Bernoulli numbers $\beta_{n}$ are defined by

$$
\frac{x}{e^{x}-1}=\sum_{n \geq 0} \frac{\beta_{n}}{n !} x^{n}
$$

We know that $\beta_{0}=1, \beta_{1}=-1 / 2$ and $\beta_{2 i+1}=0$ if $i \geq 1$. For $i \geq 1$, we set $B_{i}=(-1)^{i+1} \beta_{2 i+1}$. These numbers $B_{i}$ are are positive and also called Bernoulli numbers. By an easy calculation we obtain Hirzebruch power series

$$
h(x)=\frac{x}{\tanh x}=1+\sum_{i \geq 1} \frac{(-1)^{i+1} 2^{2 i} B_{i}}{(2 i) !} x^{2 i} .
$$

To simplify our notation, we put $a_{i}=(h(x))_{2 i}=(-1)^{i+1} 2^{2 i} B_{i} /(2 i)$ !. Then $h(x)=$ $\sum_{i \geq 0} a_{i} x^{2 i}$. The 2 -orders of these coefficients $a_{i}$ are given by the following lemma which immediately implies that $h(x)$ is in $\mathbb{Z}_{(2)}[[x]]$.

Lemma 12. $\nu_{2}\left(a_{i}\right)=\kappa_{2}(i)-1$ for all $i$.

Proof. You can prove the assertion by using the theorem of Clausen-von Staudt $\nu_{2}\left(B_{i}\right)=-1$, (see [3]). However to keep our exposition self-contained, we shall present here an alternative elementary proof. By multiplying $h(x)$ by $\sinh 2 x$, we have

$$
h(x) \sinh 2 x=x(\cosh 2 x+1) .
$$

When we take the $(2 n+1)$-th derivative $(n \geq 1)$ using the general Leibniz rule, we get

$$
\sum_{i=0}^{2 n+1}\left(\begin{array}{c}
2 n+1 \\
i
\end{array}\right) h^{(i)}(x)(\sinh 2 x)^{(2 n+1-i)}=x 2^{2 n+1} \sinh 2 x+(2 n+1) 2^{2 n} \cosh 2 x .
$$

Substituting $x=0$ we have

$$
\sum_{j=0}^{n}\left(\begin{array}{c}
2 n+1 \\
2 j
\end{array}\right) h^{(2 j)}(0) 2^{2 n+1-2 j}=(2 n+1) 2^{2 n} .
$$


Division by $2^{2 n}$ gives

$$
\sum_{j=0}^{n}\left(\begin{array}{c}
2 n+1 \\
2 j
\end{array}\right) \frac{h^{(2 j)}(0)}{2^{2 j-1}}=2 n+1
$$

We put $u_{j}=h^{(2 j)}(0) /\left(2^{2 j-1}\right)$ and since $h(0)=1$ we have

$$
\sum_{j=1}^{n}\left(\begin{array}{c}
2 n+1 \\
2 j
\end{array}\right) u_{j}=2 n-1
$$

When $n=1$, we have $u_{1}=1 / 3$ and $u_{1} \equiv 3 \bmod 4$ in $\mathbb{Z}_{(2)}$. We will show that $u_{j} \in \mathbb{Z}_{(2)}$ and $u_{j} \equiv 1 \bmod 4$ for $j \geq 2$ by induction on $j$. Suppose that $u_{j} \in \mathbb{Z}_{(2)}$ and $u_{j} \equiv 1 \bmod 4$ for $2 \leq j \leq n-1 \quad n \geq 3$. We know from the binomial expansion formula that

$$
\sum_{j=1}^{n}\left(\begin{array}{c}
2 n+1 \\
2 j
\end{array}\right)=2^{2 n}-1
$$

Subtracting this from (3), we have

$$
\sum_{j=1}^{n}\left(\begin{array}{c}
2 n+1 \\
2 j
\end{array}\right)\left(u_{j}-1\right)=2 n-2^{2 n} \equiv 2 n \quad \bmod 4 .
$$

On the other hand, from the inductive assumption we have

$$
\begin{aligned}
\sum_{j=1}^{n}\left(\begin{array}{c}
2 n+1 \\
2 j
\end{array}\right)\left(u_{j}-1\right) & \equiv 2\left(\begin{array}{c}
2 n+1 \\
2
\end{array}\right)+(2 n+1)\left(u_{n}-1\right) \bmod 4 \\
& =2 n(2 n+1)+(2 n+1)\left(u_{n}-1\right) .
\end{aligned}
$$

Thus we have

$$
2 n(2 n+1)+(2 n+1)\left(u_{n}-1\right) \equiv 2 n \bmod 4 .
$$

Hence $(2 n+1)\left(u_{n}-1\right) \equiv 0 \bmod 4$. Therefore we have $u_{n} \equiv 1 \bmod 4$. From this we have $\nu_{2}\left(h^{(2 j)}(0)\right)=2 j-1$. Finally we have

$$
\nu_{2}\left(a_{i}\right)=\nu_{2}\left(h^{(2 i)}(0)\right)-\nu_{2}((2 i) !)=(2 i-1)-\left(2 i-\kappa_{2}(2 i)\right)=\kappa_{2}(i)-1 .
$$

We shall define another formal power series

$$
g(x)=\frac{1}{8}\left(\frac{h(3 x)}{h(x)}-1\right)=\frac{1}{8}\left(\frac{3 \tanh x}{\tanh 3 x}-1\right)=\frac{\tanh ^{2} x}{3+\tanh ^{2} x} .
$$

We shall simply express this formal power series as

$$
g(x)=\sum_{i \geq 1} b_{i} x^{2 i}
$$

Since all the coefficients of the formal power series of

$$
\tanh x=\sum_{i \geq 1} \frac{(-1)^{i+1} 2^{2 i}\left(2^{2 i}-1\right) B_{i}}{(2 i) !} x^{2 i-1}
$$

belong to $\mathbb{Z}_{(2)}$, the coefficients $b_{i}$ of $g(x)$ all belong to $\mathbb{Z}_{(2)}$. About their 2-orders we have the following lemma. Remark that this fact does not follow immediately from the theorem of Clausen-von Staudt.

Lemma 13. $\nu_{2}\left(b_{i}\right)=\kappa_{2}(i)-1$ for all $i \geq 1$. 
Proof. The proof is done using a similar argument of the proof of the previous lemma.

We have

$$
g(x)=\frac{\tanh ^{2} x}{3+\tanh ^{2} x}=\frac{\sinh ^{2} x}{3 \cosh ^{2} x+\sinh ^{2} x}=\frac{\cosh 2 x-1}{4 \cosh 2 x+2},
$$

and

$$
(2 \cosh 2 x+1) g(x)=(\cosh 2 x-1) / 2 .
$$

Taking the $2 n$-th derivative $(n \geq 1)$ of both hands, we have

$$
g^{(2 n)}(x)(2 \cosh 2 x+1)+\sum_{i=1}^{2 n}\left(\begin{array}{c}
2 n \\
i
\end{array}\right) g^{(2 n-i)}(x)(2 \cosh 2 x)^{(i)}=2^{2 n-1} \cosh 2 x .
$$

Substituting $x=0$ in this expression, we have

$$
3 g^{(2 n)}(0)+\sum_{j=1}^{n}\left(\begin{array}{l}
2 n \\
2 j
\end{array}\right) 2^{2 j+1} g^{(2 n-2 j)}(0)=2^{2 n-1}
$$

Define $u_{j}=g^{(2 j)}(0) / 2^{2 j-1}$, then we have

$$
3 u_{n}+2 \sum_{j=1}^{n-1}\left(\begin{array}{l}
2 n \\
2 j
\end{array}\right) u_{n-j}=1 .
$$

By putting $n=1$ to (44), we have $u_{j}=1 / 3 \equiv 3 \bmod 4$. We shall prove that for all $u_{j} \equiv 3 \bmod 4$ for all $j$. We use induction and let us suppose that $u_{j} \equiv 3 \bmod 4$ for $j<n$. Then from (4), we have

$$
3 u_{n}+6 \sum_{j=1}^{n-1}\left(\begin{array}{l}
2 n \\
2 j
\end{array}\right) \equiv 1 \bmod 4
$$

Since $\sum_{j=1}^{n-1}\left(\begin{array}{c}2 n \\ 2 j\end{array}\right)=2^{2 n-1}-2$ is even, we have $3 u_{n} \equiv 1 \bmod 4$ and this implies that $u_{n} \equiv 3 \bmod 4$. Thus for all $j, u_{j} \equiv 3 \bmod 4$. Therefore

$$
\nu_{2}\left(b_{i}\right)=\nu_{2}\left(g^{(2 i)}(0) /(2 i) !\right)=(2 i-1)-\left(2 i-\kappa_{2}(2 i)\right)=\kappa_{2}(i)-1 .
$$

\section{The INDEX THEOREM FOR A HOMOTOPY $\mathbb{C} P(2 k)$}

Let $\eta$ be the canonical complex line bundle over $\mathbb{C} P(2 k)$ whose first Chern class $x=c_{1}(\eta)$ is generates the cohomology ring $H^{*}(\mathbb{C} P(2 k) ; \mathbb{Z})=\mathbb{Z}[x] /\left(x^{2 k+1}\right)$. If $f: M^{4 k} \rightarrow \mathbb{C} P(2 k)$ is a homotopy $\mathbb{C} P(2 k)$, then there exists a fiber homotopically trivial vector bundle $\zeta$ over $\mathbb{C} P(2 k)$ such that the tangent bundle $\tau(M)$ is stably isomorphic to the pullback of $\tau(\mathbb{C} P(2 k)) \oplus \zeta$ by $f$ :

$$
\tau(M) \stackrel{s}{\sim} f^{*}(\tau(\mathbb{C} P(2 k)) \oplus \zeta)
$$

Using Hirzebruch's index thorem, we see that

$$
\operatorname{Index}(M)=\langle\mathcal{L}(M),[M]\rangle=\left\langle\mathcal{L}(\zeta) h(x)^{2 k+1},[\mathbb{C} P(2 k)]\right\rangle,
$$

where $\mathcal{L}$ denotes Hirzebruch's $L$-class $\sum_{j} L_{j}$ associated to the power series $h(x)$. Since $\delta(M) x^{2}$ coincides with $p_{1}(\zeta)$, we have to examine the Pontrjagin class of $\zeta$ when $\operatorname{Index}(M)=1$ holds.

Let $\omega \in \widetilde{K O}(\mathbb{C} P(2 k))$ denote the realification of $\eta-1_{\mathbb{C}} \in \widetilde{K}(\mathbb{C} P(2 k))$. It is known that $\widetilde{K O}(\mathbb{C} P(2 k))$ is a free abelian group generated by $\omega^{j}(j=1,2, \ldots, k)$ $([10])$. The real Adams operation on $\omega$ is given by the formula

$$
\psi_{\mathbb{R}}^{j}(\omega)=T_{j}(\omega),
$$


where $T_{j}(z)$ is a polynomial of $z$ having degree $j$ characterized by the property

$$
T_{j}\left(t+t^{-1}-2\right)=t^{j}+t^{-j}-2 .
$$

Since the coefficient of $z^{j}$ in $T_{j}(z)$ is one, we may take $\psi_{\mathbb{R}}^{j}(\omega)=T_{j}(\omega) \quad(1 \leq j \leq k)$ as generators of $\widetilde{K O}(\mathbb{C} P(2 k))$.

According to the solution of the Adams-conjecture, the kernel of the $J$-map coincides with Image $\left(\psi_{\mathbb{Q}}^{3}-1\right)$ when localized at 2 . Therefore when we put $\zeta_{j}=$ $\left(\psi_{\mathbb{R}}^{3}-1\right) \psi_{\mathbb{R}}^{j}(\omega)$, the fiber homotopically trivial vector bundle $\zeta$ can be written as

$$
q \zeta=n_{1} \zeta_{1}+n_{2} \zeta_{2}+\cdots+n_{k} \zeta_{k},
$$

for some integers $n_{1}, n_{2}, \ldots, n_{k}$ and an odd integer $q$. Therefore we may write

$$
\zeta=m_{1} \zeta_{1}+m_{2} \zeta_{2}+\cdots+m_{k} \zeta_{k}
$$

where $m_{j}, m_{2}, \ldots, m_{k}$ belong to $\mathbb{Z}_{(2)}$. We first calculate the total Pontrjagin class of $\psi_{\mathbb{R}}^{j}(\omega)$. We first note that

$$
\begin{aligned}
\psi_{\mathbb{R}}^{j}(\omega) \otimes \mathbb{C} & =\psi_{\mathbb{C}}^{j}(\omega \otimes \mathbb{C})=\psi_{\mathbb{C}}^{j}\left(\eta+\bar{\eta}-2_{\mathbb{C}}\right) \\
& =\psi_{\mathbb{C}}^{j}(\eta)+\psi_{\mathbb{C}}^{j}(\bar{\eta})-2_{\mathbb{C}}=\eta^{j}+\bar{\eta}^{j}-2_{\mathbb{C}},
\end{aligned}
$$

whose Chern class is equal to $(1+j x)(1-j x)=1-j^{2} x^{2}$. Therefore the total Pontrjagin classes are given by

$$
\begin{gathered}
p\left(\psi_{\mathbb{R}}^{j}(\omega)\right)=1+j^{2} x^{2}, \\
p\left(\zeta_{j}\right)=p\left(\psi_{\mathbb{R}}^{3 j}(\omega)-\psi_{\mathbb{R}}^{j}(\omega)\right)=\frac{1+(3 j)^{2} x^{2}}{1+j^{2} x^{2}},
\end{gathered}
$$

and

$$
p(\zeta)=\prod_{j=1}^{k} p\left(\psi_{\mathbb{R}}^{j}(\omega)\right)^{m_{j}}=\prod_{j=1}^{k}\left(\frac{1+(3 j)^{2} x^{2}}{1+j^{2} x^{2}}\right)^{m_{j}} .
$$

From this we have

$$
p_{1}(\zeta)=8 \sum_{j=1}^{k} j^{2} m_{j}
$$

The $\mathcal{L}$ class of $\zeta$ is written as

$$
\mathcal{L}(\zeta)=\prod_{j=1}^{k} \mathcal{L}\left(\zeta_{j}\right)^{m_{j}}=\prod_{j=1}^{k}\left(\frac{h(3 j x)}{h(j x)}\right)^{m_{j}}=\prod_{j=1}^{k}(1+8 g(j x))^{m_{j}} .
$$

We calculate the index of $M^{2 k}$ :

$$
\begin{aligned}
& \text { Index }(M)=\left\langle\mathcal{L}(\zeta) h(x)^{2 k+1},[\mathbb{C} P(2 k)]\right\rangle \\
& =\left(\mathcal{L}(\zeta) h(x)^{2 k+1}\right)_{2 k}=\left(\prod_{j=1}^{k}(1+8 g(j x))^{m_{j}} h(x)^{2 k+1}\right)_{2 k} \\
& =1+8 \sum_{j=1}^{k} m_{j}\left(g(j x) h(x)^{2 k+1}\right)_{2 k} \\
& \quad+\sum_{s \geq 2} 8^{s} \sum_{i_{1}+\cdots+i_{k}=s}\left(\begin{array}{c}
m_{1} \\
i_{1}
\end{array}\right) \cdots\left(\begin{array}{c}
m_{k} \\
i_{k}
\end{array}\right)\left(g(x)^{i_{1}} g(2 x)^{i_{2}} \cdots g(k x)^{i_{k}} h(x)^{2 k+1}\right)_{2 k},
\end{aligned}
$$

where $(f(x))_{j}$ denotes the coefficient of $x^{j}$ in the formal power series $f(x)$. We shall use the following notations:

$$
C\left(j_{1}, j_{2}, \cdots, j_{s}\right)=\left(g\left(j_{1} x\right) g\left(j_{2} x\right) \cdots g\left(j_{s} x\right) h(x)^{2 k+1}\right)_{2 k},
$$




$$
D\left(i_{1}, i_{2}, \ldots, i_{k}\right)=C(\underbrace{1, \ldots, 1}_{i_{1}}, \underbrace{2, \ldots, 2}_{i_{2}}, \ldots, \underbrace{k, \ldots, k}_{i_{k}}) .
$$

Then we have

$$
\operatorname{Index}(M)=1+8 \sum_{j=1}^{k} m_{j} C(j)+\sum_{s \geq 2} 8^{s} \sum_{i_{1}+\cdots+i_{k}=s}\left(\begin{array}{c}
m_{1} \\
i_{1}
\end{array}\right) \cdots\left(\begin{array}{c}
m_{k} \\
i_{k}
\end{array}\right) D\left(i_{1}, \ldots, i_{k}\right) .
$$

Since $\operatorname{Index}(M)=1$, we have

$$
\sum_{j=1}^{k} m_{j} C(j)+\sum_{s \geq 2} 8^{s-1} \sum_{i_{1}+\cdots+i_{k}=s}\left(\begin{array}{c}
m_{1} \\
i_{1}
\end{array}\right) \cdots\left(\begin{array}{c}
m_{k} \\
i_{k}
\end{array}\right) D\left(i_{1}, \ldots, i_{k}\right)=0 .
$$

Our target is to show that $p_{1}(\zeta)$ is divisible by 16 from the condition (5). This is equivalent to the claim that $\sum_{j=1}^{k} j^{2} m_{j}$ is even. This is also equivalent to $\sum_{j: \text { odd }} m_{j}$ is even.

\section{Lemma 14.}

$$
\begin{aligned}
\left(g(x)^{s} h(x)^{2 k+1}\right)_{2 k} & =C(\underbrace{1, \ldots, 1}_{s})=D(s, 0, \ldots, 0)=\left(\frac{1}{(3+x)^{s}(1-x)}\right)_{k-s} \\
& =\frac{1}{4^{s}}\left(\frac{1}{1-x}+\frac{1}{3+x}+\frac{4}{(3+x)^{2}}+\cdots+\frac{4^{s-1}}{(3+x)^{s}}\right)_{k-s} \\
& =\frac{1}{4^{s} 3^{k}}\left(3^{k}+(-1)^{k-s} \sum_{i=0}^{s-1}\left(\begin{array}{c}
k-s+i \\
i
\end{array}\right) 3^{s-1-i} 4^{i}\right) .
\end{aligned}
$$

Proof.

$$
\begin{gathered}
\left(g(x)^{s} h(x)^{2 k+1}\right)_{2 k}=\left(\left(\frac{\tanh ^{2} x}{3+\tanh ^{2} x}\right)^{s}\left(\frac{x}{\tanh x}\right)^{2 k+1}\right)_{x^{2 k}} \\
=\operatorname{Res}_{x}\left(\left(\frac{\tanh ^{2} x}{3+\tanh ^{2} x}\right)^{s} \frac{1}{\tanh ^{2 k+1} x}\right)
\end{gathered}
$$

by putting $y=\tanh x$,

$$
\begin{aligned}
& =\operatorname{Res}_{y}\left(\left(\frac{y^{2}}{3+y^{2}}\right)^{2} \frac{1}{y^{2 k+1}\left(1-y^{2}\right)}\right) \\
& =\operatorname{Res}_{y}\left(\frac{1}{y^{2 k+1-2 s}\left(3+y^{2}\right)^{s}\left(1-y^{2}\right)}\right) \\
& =\left(\frac{1}{\left(3+y^{2}\right)^{s}\left(1-y^{2}\right)}\right)_{y^{2 k-2 s}}=\left(\frac{1}{(3+x)^{s}(1-x)}\right)_{x^{k-s}} .
\end{aligned}
$$

By induction we can show that

$$
\frac{1}{(3+x)^{s}(1-x)}=\frac{1}{4^{s}}\left(\frac{1}{1-x}+\frac{1}{3+x}+\frac{4}{(3+x)^{2}}+\cdots+\frac{4^{s-1}}{(3+x)^{s}}\right) .
$$


From this we have

$$
\begin{aligned}
& \left(\frac{1}{(3+x)^{s}(1-x)}\right)_{k-s} \\
& =\frac{1}{4^{s}}\left(1+\left(-\frac{1}{3}\right)^{k-1}\left(\frac{1}{3}+\frac{4}{3^{2}}\left(\begin{array}{c}
k-s+1 \\
1
\end{array}\right)+\frac{4^{2}}{3^{3}}\left(\begin{array}{c}
k-s+2 \\
2
\end{array}\right)+\cdots+\frac{4^{s-1}}{3^{s}}\left(\begin{array}{c}
k-1 \\
s-1
\end{array}\right)\right)\right) \\
& =\frac{1}{4^{s} 3^{k}}\left(3^{k}+(-1)^{k-s} \sum_{i=0}^{s-1}\left(\begin{array}{c}
k-s+i \\
i
\end{array}\right) 3^{s-1-i} 4^{i}\right) .
\end{aligned}
$$

To simplify our notation, from now on we shall denote $\nu_{2}(k)$ by $r$. As a special case $s=1$, we have the following corollary.

Corollary 15. (a) $C(1)=\left(3^{k}-(-1)^{k}\right) /\left(4 \cdot 3^{k}\right)$, and $(\mathrm{b}) \nu_{2}(C(1))=r$.

Proof. (a) follows from the previous lemma. (b) is a result of (a) using Lemma 10 (c).

Proposition 16. If $j$ is odd, then $\nu_{2}(C(j))=r$.

Proof. The case for $j=1$ is given in Corollary 15. For any other odd number $j$, we have

$$
\begin{aligned}
& C(j)-C(1)=\left((g(j x)-g(x)) h(x)^{2 k+1}\right)_{2 k}=\left((g(j x)-g(x)) h(x) h(x)^{2 k}\right)_{2 k} \\
& =\left(\sum_{i_{1} \geq 1} b_{i_{1}}\left(j^{2 i_{1}}-1\right) x^{2 i_{1}} \sum_{i_{2} \geq 0} a_{i_{2}} x^{2 i_{2}} \sum_{i_{3} \geq 0} a_{i_{3}}^{(2 k)} x^{2 i_{3}}\right)_{2 k}=\sum_{i_{1}+i_{2}+i_{3}=k, i_{1} \geq 1}\left(j^{2 i_{1}}-1\right) b_{i_{1}} a_{i_{2}} a_{i_{3}}^{(2 k)} .
\end{aligned}
$$

Here we put $U=\left(j^{2 i_{1}}-1\right) b_{i_{1}} a_{i_{2}} a_{i_{3}}^{(2 k)}$. From Lemma 10 (b) we have

$$
\nu_{2}\left(j^{2 i_{1}}-1\right) b_{i_{1}}=\nu_{2}\left(j^{2}-1\right)+\nu_{2}\left(i_{1}\right)+\kappa_{2}\left(i_{1}\right)-1 \geq \nu_{2}\left(i_{1}\right)+\kappa_{2}\left(i_{1}\right)+2 .
$$

From Lemma 13, we have

$$
\nu_{2}\left(a_{i_{2}}\right)=\kappa_{2}\left(i_{2}\right)-1
$$

From Lemma 8, we have

$$
\nu_{2}\left(a_{i_{3}}^{(2 k)}\right) \geq \nu_{2}(2 k)-\nu_{2}\left(i_{3}\right)=r+1-\nu_{2}\left(i_{3}\right) .
$$

Thus we have $\nu_{2}(U) \geq \nu_{2}\left(i_{1}\right)+\kappa_{2}\left(i_{1}\right)+\kappa_{2}\left(i_{2}\right)+1+\nu_{2}\left(a_{i_{3}}^{(2 k)}\right)$. From this, we have $\nu_{2}(U) \geq \nu_{2}\left(i_{1}\right)+\kappa_{2}\left(i_{1}\right)+\kappa_{2}\left(i_{2}\right)+1+\nu_{2}\left(a_{i_{3}}^{(2 k)}\right) \geq \nu_{2}\left(k-i_{3}\right)+2+\max \left(r+1-\nu_{2}\left(i_{3}\right), 0\right)$. If $i_{3}=0$, from Lemma 11, we have $\nu_{2}(U) \geq \nu_{2}\left(i_{1}+i_{2}\right)+2=r+2$. If $i_{3} \geq 1$ and $\nu_{2}\left(i_{3}\right) \leq r$, then since $\nu_{2}\left(k-i_{3}\right) \geq \nu_{2}\left(i_{3}\right)$, we have $\nu_{2}(U) \geq r+3$. If $\nu_{2}\left(i_{3}\right)>r$, then since $\nu_{2}\left(k-i_{3}\right)=r$, we have $\nu_{2}(U) \geq r+2$. Therefore we always have $\nu_{2}(U) \geq r+2$. This shows that $\nu_{2}(C(j)-C(1)) \geq r+2$. Therefore we have $\nu_{2}(C(j))=r$.

Proposition 17. Let $j$ be an even natural number. Then $\nu_{2}(C(j)) \geq r+1$.

Proof. As in the proof of Proposition 16, we have

$$
C(j)=\sum_{i_{1}+i_{2}+i_{3}=k, i_{1} \geq 1} j^{2 i_{1}} b_{i_{1}} a_{i_{2}} a_{i_{3}}^{(2 k)} .
$$

Put $B=j^{2 i_{1}} b_{i_{1}} a_{i_{2}} a_{i_{3}}^{(2 k)}$. Since $\nu_{2}\left(j^{2 i_{1}}\right) \geq 2 i_{1}, \nu_{2}\left(b_{i_{1}}\right)=\kappa_{2}\left(i_{1}\right)-1, \nu_{2}\left(a_{i_{2}}\right)=$ $\kappa_{2}\left(i_{2}\right)-1$ and $\nu_{2}\left(a_{i_{3}}^{(2 k)}\right) \geq \max \left(r+1-\nu_{2}\left(i_{3}\right), 0\right)$, we have

$$
\begin{aligned}
\nu_{2}(B) & \geq 2 i_{1}+\kappa_{2}\left(i_{1}\right)+\kappa_{2}\left(i_{2}\right)-2+\max \left(r+1-\nu_{2}\left(i_{3}\right), 0\right) \\
& \geq 2 i_{1}+\nu_{2}\left(k-i_{3}\right)-\nu_{2}\left(i_{1}\right)-1+\max \left(r+1-\nu_{2}\left(i_{3}\right), 0\right) .
\end{aligned}
$$


If $i_{3}=0$, then since $i_{1} \geq \nu_{2}\left(i_{1}\right)+1$, we have

$$
\nu_{2}(B) \geq 2 i_{1}+r-\nu_{2}\left(i_{1}\right)-1 \geq r+i_{1} \geq r+1 .
$$

If $i_{3} \geq 1$ and $\nu_{2}\left(i_{3}\right) \leq r$, then we have $\nu_{2}\left(k-i_{3}\right) \geq \nu_{3}\left(i_{3}\right)$. Hence we have $\nu_{2}(B) \geq 2 i_{1}+\nu_{2}\left(i_{3}\right)-\nu_{2}\left(i_{1}\right)-1+\left(r+1-\nu_{2}\left(i_{3}\right)\right)=r+2 i_{1}-\nu_{2}\left(i_{1}\right) \geq r+1+\left(i_{1}-\nu_{2}\left(i_{1}\right)\right) \geq r+2$.

Finally if $i_{3} \geq 1$ and $\nu_{2}\left(i_{3}\right)>r$, then we have

$$
\nu_{2}(B) \geq 2 i_{1}+r-\nu_{2}\left(i_{1}\right)-1 \geq i_{1}+r \geq r+1 .
$$

Thus in all cases, we have $\nu_{2}(B) \geq r+1$. This proves our assertion.

Lemma 18. $\nu_{2}(C(\underbrace{1, \ldots, 1}_{s})) \geq r+2-2 s$.

Proof. The assertion is true for $s=1$ by Corollary 15 . So we assume that $s \geq 2$. From Lemma 14 we have

$$
\begin{aligned}
C(\underbrace{1, \cdots, 1}_{s}) & =\frac{1}{4^{s} 3^{k}}\left(3^{k}+(-1)^{k-s} \sum_{i=0}^{s-1}\left(\begin{array}{c}
k-s+i \\
i
\end{array}\right) 3^{s-1-i} 4^{i}\right) \\
& =\frac{1}{4^{s} 3^{k}}\left(3^{k}+(-1)^{k-s} \sum_{i=0}^{s-1} \frac{(k-s+1)(k-s+2) \cdots(k-s+i)}{i !} 3^{s-1-i} 4^{i}\right) \\
& =\frac{1}{4^{s} 3^{k}}\left(3^{k}+(-1)^{k-s} \sum_{i=0}^{s-1} \frac{3^{s-1-i} 4^{i}}{i !}(k-s+1)(k-s+2) \cdots(k-s+i)\right) .
\end{aligned}
$$

Define a polynomial with variable $k$ :

$$
w(k)=\sum_{i=1}^{s-1} \frac{3^{s-1-i} 4^{i}}{i !}(k-s+1)(k-s+2) \cdots(k-s+i) .
$$

We have

$$
\nu_{2}\left(\frac{3^{s-1-i} 4^{i}}{i !}\right)=2 i-\left(i-\kappa_{2}(i)\right)=i+\kappa_{2}(i) \geq 2 .
$$

Therefore $w(k)$ is a polynomial in $k$ with coefficients in $4 \mathbb{Z}_{(2)}$. As for the constant term $w(0)$, we have

$$
\begin{aligned}
w(0) & =\sum_{i=1}^{s-1} \frac{3^{s-1-i} 4^{i}}{i !}(-s+1)(-s+2) \cdots(-s+i) \\
& =\sum_{i=1}^{s-1} \frac{(s-1)(s-2) \cdots(s-i)}{i !} 3^{s-1-i}(-4)^{i} \\
& =\sum_{i=1}^{s-1}\left(\begin{array}{c}
s-1 \\
i
\end{array}\right) 3^{s-1-i}(-4)^{i}=(3+(-4))^{s-1}-3^{s-1}=(-1)^{s-1}-3^{s-1} .
\end{aligned}
$$

Therefore we have

$$
\begin{aligned}
C(\underbrace{1, \cdots, 1}_{s}) & =\frac{1}{4^{s} 3^{k}}\left(3^{k}+(-1)^{k-s}\left(3^{s-1}+w(k)\right)\right) \\
& =\frac{1}{4^{s} 3^{k}}\left(3^{k}+(-1)^{k-s}\left(3^{s-1}+w(0)\right)+(-1)^{k-s}(w(k)-w(0))\right) \\
& =\frac{1}{4^{s} 3^{k}}\left(3^{k}-(-1)^{k}+(-1)^{k-s}(w(k)-w(0))\right) .
\end{aligned}
$$

By Lemma 10, $\nu_{2}\left(3^{k}-(-1)^{k}\right)=r+2$. As a polynomial in $k$, all the coefficients of $w(k)-w(0)$ in have 2 -orders at least 2. Therefore $\nu_{2}(w(k)-w(0)) \geq r+2$. This proves that $\nu_{2}(C(1, \ldots, 1)) \geq r+2-2 s$. 
Lemma 19. Let $j_{1}, j_{2}, \ldots, j_{s}$ and $j_{1}^{\prime}$ be odd natural numbers. Then $\nu_{2}\left(C\left(j_{1}, j_{2}, \ldots, j_{s}\right)-\right.$ $\left.C\left(j_{1}^{\prime}, j_{2}, \ldots, j_{s}\right)\right) \geq r+3-s$.

Proof. We have

$$
\begin{aligned}
C\left(j_{1}, j_{2}, \ldots, j_{s}\right)-C\left(j_{1}^{\prime}, j_{2}, \ldots, j_{s}\right) & \\
\quad= & \sum_{i_{1}+\cdots+i_{s}+i_{s+1}+l=k}\left(j_{1}^{2 i_{1}}-j_{1}^{\prime 2 i_{1}}\right) j_{2}^{2 i_{2}} \cdots j_{s}^{2 i_{s}} b_{i_{1}} b_{i_{2}} \cdots b_{i_{s}} a_{i_{s+1}} a_{l}^{(2 k)} .
\end{aligned}
$$

We put $V=\left(j_{1}^{2 i_{1}}-j_{1}^{\prime 2 i_{1}}\right) j_{2}^{2 i_{2}} \cdots j_{s}^{2 i_{s}} b_{i_{1}} b_{i_{2}} \cdots b_{i_{s}} a_{i_{s+1}} a_{l}^{(2 k)}$. From Lemma 10, we have

$$
\nu_{2}\left(j^{2 i_{1}}-j_{1}^{\prime 2 i_{1}}\right) \geq 3+\nu_{2}\left(i_{1}\right) .
$$

From Lemmas 11, 12 and 13, we have

$$
\nu_{2}\left(b_{i_{1}} \cdots b_{i_{s}} a_{i_{s+1}}\right)=\kappa_{2}\left(i_{1}\right)+\cdots+\kappa_{2}\left(i_{s+1}\right)-(s+1) \geq \nu_{2}(k-l)-\nu_{2}\left(i_{1}\right)-s .
$$

Hence we have

$$
\nu_{2}(V) \geq \nu_{2}(k-l)+3-s+\nu_{2}\left(a_{l}^{(2 k)}\right) .
$$

If $l=0$, then we have

$$
\nu_{2}(V) \geq r+3-s .
$$

If $l \geq 1$ and $\nu_{2}(l) \leq r$, then from Corollary 8 we have

$$
\nu_{2}(V) \geq \nu_{2}(l)+3-s+\left(r+1-\nu_{2}(l)\right)=r+4-s .
$$

If $l \geq 1$ and $\nu_{2}(l)>r$, then we have

$$
\nu_{2}(V) \geq r+3-s .
$$

Therefore $\nu_{2}(V) \geq r+3-s$ holds.

Corollary 20. If $j_{1}, \ldots, j_{s}$ are all odd, then we have $\nu_{2}\left(C\left(j_{1}, \ldots, j_{s}\right)\right) \geq r+2-2 s$.

Proof. From Lemma 19, we have $\nu_{2}\left(C\left(j_{1}, \ldots, j_{s}\right)-C(1, \ldots, 1)\right) \geq r+3-s$. On the other we know from Corollary 18 that $C(1, \ldots, 1)$ satisfies $\nu_{2}(C(1, \ldots, 1)) \geq$ $r+2-2 s$. Since $r+3-s>r+2-2 s$ holds, we have $\nu_{2}\left(C\left(j_{1}, \ldots, j_{s}\right)\right) \geq r+2-2 s$.

Lemma 21. If there exists at least one even number in $j_{1}, \ldots, j_{s}$, then we have

$$
\nu_{2}\left(C\left(j_{1}, \ldots, j_{2}\right)\right) \geq r+2-s .
$$

Proof. Without loss of generality, we may assume that $j_{1}$ is even. We have

$$
C\left(j_{1}, \ldots, j_{s}\right)=\sum_{i_{1}+\cdots+i_{s+1}+l=k} j_{1}^{2 i_{1}} \cdots j_{s}^{2 i_{s}} b_{i_{1}} \cdots b_{i_{s}} a_{i_{s+1}} a_{l}^{(2 k)} .
$$

We put $U=j_{1}^{2 i_{1}} \cdots j_{s}^{2 i_{s}} b_{i_{1}} \cdots b_{i_{s}} a_{i_{s+1}} a_{l}^{(2 k)}$. Since $j_{1}$ is even, we have

$$
\nu_{2}\left(j_{1}^{2 i_{1}} \cdots j_{s}^{2 i_{s}}\right) \geq 2 i_{1},
$$

and

$$
\nu_{2}\left(b_{i_{1}} \cdots b_{i_{s}} a_{i_{s+1}}\right)=\kappa_{2}\left(i_{1}\right)+\cdots+\kappa_{2}\left(i_{s+1}\right)-(s+1) \geq \nu_{2}(k-l)-\nu_{2}\left(i_{1}\right)-s .
$$

Hence we have

$$
\nu_{2}(U) \geq 2 i_{1}+\nu_{2}(k-l)-\nu_{2}\left(i_{1}\right)-s+\nu_{2}\left(a_{l}^{(2 k)}\right) .
$$

If $l=0$, then we have

$$
\nu_{2}(U) \geq 2 i_{1}+r-\nu_{2}\left(i_{1}\right)-s \geq r+2-s .
$$

If $i \geq 1$ and $\nu_{2}(l) \leq r$, then we have

$$
\nu_{2}(U) \geq 2 i_{1}+\nu_{2}(l)-\nu_{2}\left(i_{1}\right)-s+\left(r+1-\nu_{2}(l)\right) \geq r-s+3 .
$$

If $i \geq 1$ and $\nu_{2}(l)>r$, then we have

$$
\nu_{2}(U) \geq 2 i_{1}+r-\nu_{2}\left(i_{1}\right)-s \geq r+2-s .
$$


This shows that $\nu_{2}(U) \geq r-s+2$ always holds. From this we conclude that

$$
\nu_{2}\left(C\left(j_{1}, \ldots, j_{s}\right)\right) \geq r+2-s .
$$

Combining Corollary 20 and Lemma 21, we have the following proposition.

Proposition 22. If $s \geq 2$, then for any natural numbers $j_{1}, j_{2}, \ldots, j_{s}$, we have

$$
\nu_{2}\left(C\left(j_{1}, j_{2}, \ldots, j_{s}\right)\right) \geq r+2-2 s .
$$

\section{Proof of the main theorem}

If $M^{4 k}$ is a homotopy $\mathbb{C} P(2 k)$, we obtained the relation (5). From Lemma 22, we have

$$
\nu_{2}\left(8^{s-1} D\left(i_{1}, \ldots, i_{s}\right)\right) \geq 3(s-1)+r+2-2 s=r+s-1 \geq r+1 .
$$

Thus from (5), we have

$$
\sum_{j=1}^{k} m_{j} C(j) \equiv 0 \quad \bmod 2^{r+1} .
$$

From Proposition [16, we know that when $j$ is odd then $C(j) \equiv 1 \bmod 2^{r+1}$. And from Proposition $\left[16\right.$, we have $C(j) \equiv 0 \bmod 2^{r+1}$ when $j$ is even. Therefore we have proved that $\sum_{1 \leq j \leq k, j \text { :odd }} m_{j}$ is even. This shows that first Pontrjagin class of $\zeta$

$$
p_{1}(\zeta)=8 \sum_{j=1}^{k} j^{2} m_{j}
$$

is divisible by 16 . This proves our main theorem.

\section{REFERENCES}

[1] BROWDER, W., Surgery and the theory of differentiable transformation groups, In: Proceedings of the Conference on Transformation Groups, New Orleans 1967, Springer-Verlag, 1968, pp. 1-46.

[2] BRUMFIEL, G., Homotopy equivalences of almost smooth manifolds, Comment. Math. Helv. 46 (1971), 381-407.

[3] HARDY, G.H. and WRIGHT, E.M., An introduction to the theory of numbers, $5^{\text {th }}$ Edition, Oxford Univ. Press, 1979.

[4] KITADA, Y., On the first Pontrjagin class of homotopy complex projective spaces, Math. Slovaca, 62(2012), No. 3, 551-566.

[5] MADSEN, I., JAMES, R. and MILGRAM, J., The classifying spaces for surgery and cobordism of manifolds, Annals of Mathematics Studies, Princeton Univ. Press, 1979.

[6] MASUDA, M. and TSAI, Y-D., Tangential representations of cyclic group actions on homotopy complex projective spaces, Osaka J. Math., 23 (1985), 907-919.

[7] MONTGOMERY, D. and YANG, C.T., Differentiable actions on homotopy seven spheres II, In: Proceedings of the Conference on Transformation Groups, New Orleans 1967, SpringerVerlag, 1968, pp. 125-134.

[8] MONTGOMERY, D. and YANG, C.T., Free differentiable actions on homotopy spheres, In: Proceedings of the Conference on Transformation Groups, New Orleans 1967, SpringerVerlag, 1968, pp. 175-192.

[9] QUILLEN, D., The Adams conjecture, Topology 10 (1971), 67-80.

[10] SANDERSON, B.J., Immersions and embeddings of projective spaces, Proc. London Math. Soc., 3(1964), 137-153.

[11] SPIVAK, M., Spaces satisfying Poincaré duality, Top. 6(1967), 77-101.

(Y. Kitada) Yokohama National University E-mail address, Y. Kitada: ykitada@ynu.ac.jp

(M. Nagura) Yokohama National University E-mail address, M. Nagura: maki@ynu.ac.jp 